Historic, Archive Document

Do not assume content reflects current scientific knowledge, policies, or practices. 



\section{GENERAL PRICE LIST OF \\ Fruit, Shade and Ornamental Trees, Hardy Plants and Shrubs}

We can furnish all varieties usually catalogued. The following are the most desirable :

\section{APPLES.}

Summer Varieties.

Early Harvest-Pale yellow, fine flavor; August.

Red Astrachan — Large, crimson, juicy; July.

Autumn Varieties.

Gravenstein-Large, striped, fine quality; October.

Rambo-Yellow, streaked red; December.

\section{Winter Varieties.}

Baldwin-Large, dark red, productive.

Gano-Deep red, fine quality.

Golden Russet-Dull russet, crisp, juicy.

Wine Sap-Deep red, sub-acid.

5 to 7 feet high.

\section{CRAB APPLES.}

Hyslop-Deep crimson.

Transcendant-Skin yellow, striped red. 40 c each, $\$ 4.00$ per dozen.

\section{CHERRIES.}

Sweet.

Black Tartarian - Large, purplish black, juicy.

Governor Wood-Large, light red, productive.

\section{Sour.}

May Duke-Large, red, an excellent variety.

Morello-Dark red, rich and good. 5 to 7 feet high.

50 c each, $\$ 5.00$ per dozen.

\section{PEACHES.}

Champion-White, with red cheeks, freestone; August.

Elberta-Large, yellow, with red cheeks, firm, juicy; September

Crawford, Late-Yellow, freestone; late September. Heath Cling-Large, white flesh; October.
5 to 6 feet.

25c each, $\$ 2.50$ per dozen.

\section{PLUMS.}

Abundance-Large, yellow, good quality. Damson-Small, oval fruit, good quality.

Shippers' Pride-Large, purple, juicy and sweet.

Red June-Large, red, vigorous grower. 5 to 7 feet.

50 c each, $\$ 5.00$ per dozen.

\section{PEARS.}

Bartlett-Large, clear, yellow, juicy.

Flemish Beauty-Large, rich and fine.

Seckel-Small, yellowish brown, good.

Kieffer-Large, good keeper.

5 to 7 feet.

50 c each, $\$ 5.00$ per dozen.

\section{QUINCE.}

Orange-Large, yellow, fine quality.

Meech's Prolific - Yellow, flesh fragrant and tender.

3 to 5 feet.

50 c each, $\$ 5.00$ per dozen.

\section{GRAPES.}

Two-year-old Stock.

Black or Purple Grapes.

Campbell's Early-Bunch and berry large, sweet and juicy, $25 \mathrm{c}$ ea., $\$ 2.50$ doz.

Concord-Large, hardy and productive, 10 c ea., $\$ 1.00$ doz.

Worden-Larger than Concord, excellent flavor, 15 c ea., $\$ 1.50$ doz.

Eaton-Bunch and berry very large, finest quality, $25 \mathrm{c}$ ea., $\$ 2.50 \mathrm{doz}$.

\section{Red Grapes.}

Brighton-Large berry and bunch, early, $15 \mathrm{c}$ ea., $\$ 1.50$ doz.

Catawba-Sweet, rich, musky flavor, 10c ea., $\$ 1.00$ doz.

Delaware-Small, light red, thin skin, 15c ea., $\$ 1.50$ doz.

\section{White or Yellow Grapes.}

Niagara-Light, greenish white, hardy, $15 \mathrm{c}$ ea., $\$ 1.50 \mathrm{doz}$.

Pocklington-Large, golden yellow berry, $15 \mathrm{c}$ ea., $\$ 1.50$ doz. 


\section{GOOSEBERRIES.}

Downing-Large, pale green, excellent, 20 c ea., $\$ 2.00$ doz.

Industry - Dark red when ripe, unsurpassed, $20 \mathrm{c}$ ea., $\$ 2.00 \mathrm{doz}$.

\section{CURRANTS}

Black Champion-Large, excellent quality, strong grower, $15 \mathrm{c}$ ea., $\$ 1.50$ doz.

Perfection - The best, bright red, good bearer, 20 c ea., $\$ 2.00$ doz.

White Grape-Excellent, large, white, 10c ea., $\$ 1.00$ doz.

\section{RASPBERRIES.}

50 c doz., $\$ 2.50$ per 100 .

Cumberland - Large, healthy, vigorous grower. Black cap.

Cuthbert - Large, rich, crimson, big yielder.

\section{BLACKBERRIES.}

50 c doz., $\$ 3.50$ per 100 .

Early Harvest-Early dwarf grower, fruit medium size.

Eldorado-Large berry, in large clusters, ripens evenly.

Snyder-Medium size, sweet and juicy.

\section{DEWBERRY.}

50 c doz., $\$ 3.00$ per 100 .

Lucretia-Trailing vine, extra large berry.

\section{STRAWBERRIES.}

25 c doz., $\$ 1.00$ per 100 .

Brandywine-Fine, large, late, excellent quality.

Gandy-Good, late, large berry.

Jessie-Large, dark red.

Glen Mary-Sweet, rich, delicious.

\section{SHADE AND ORNAMENTAL TREES.}

All trees listed are 8 to 10 feet high unless noted.

Ash-American, \$1.00.

Ash-European, \$1.00.

Beech-Purple-leaved, 4 to $5 \mathrm{ft}$., $\$ 1.25$.

Birch-White, \$1.00.

Catalpa Speciosa-(Indian Cigar), 75 c.

Crab Apple-Double, flowering, 4 to 5 feet, $\$ 1.00$.

Dogwood-White, flowering, 5 to $6 \mathrm{feet}$, $\$ 1.00$.

Dogwood-Red flowering, 2 to $3 \mathrm{ft}$., $\$ 1.25$.

Elm-American, $75 \mathrm{c}$.
Ginkgo-\$1.00.

Horse Chestnut-White, 6 to $7 \mathrm{ft}$., $\$ 1.50$.

Horse Chestnut-Red, 4 to $5 \mathrm{ft} ., \$ 1.25$.

Horse Chestnut-Buckeye, 3 to $4 \mathrm{ft}$., $75 \mathrm{c}$.

Linden-American, $\$ 1.00$.

Maple-Norway, $\$ 1.00$.

Maple-Sugar, $\$ 1.25$.

Maple_Soft, $75 \mathrm{c}$.

Oak-Pin, $\$ 1.50$.

Oak-Scarlet, $\$ 1.75$.

Oak-White, $\$ 1.75$.

Peach-Flowering, red or white, 3 to 4 feet, $50 \mathrm{c}$.

Poplar-Carolina, 60c.

Poplar-Lombardy, 60 c.

Plum-Purple leaf, 5 to 6 feet, $75 \mathrm{c}$.

Sycamore-American, $75 \mathrm{c}$.

Sycamore-European, $\$ 1.00$.

Sweet Gum, \$1.50.

Tulip Tree, $\$ 1.00$.

\section{WEEPING TREES.}

Catalpa-Umbrella Tree, 6 to $8 \mathrm{ft}$., $\$ 1.50$.

Weeping Mulberry-5 to $6 \mathrm{ft}$., $\$ 1.50$.

Willow-Babylonica, $75 \mathrm{c}$.

\section{EVERGREENS.}

American Arborvitae-2 to $3 \mathrm{ft} ., 60 \mathrm{c}$.

Chinese Arborvitae.

Dwarf Golden -1 to 2 feet, $75 \mathrm{c}$.

Balsam Fir - 2 to 3 feet, $75 \mathrm{c}$.

Hemlock Spruce-18 to 24 inches, $\$ 1.00$.

Irish Juniper -2 to 3 feet, $75 \mathrm{c}$.

White Pine-2 to 3 feet, $75 \mathrm{c}$.

Dwarf Mogho Pine-12 to 18 inches, $75 \mathrm{c}$.

Koster's Blue Spruce -2 to 3 feet, $\$ 3.00$.

Norway Spruce -2 to 3 feet, $50 \mathrm{c}$.

Bush Boxwood-1 2 to 20 inches, $50 \mathrm{c}$.

Pyramid Boxwood-2 to $3 \mathrm{ft}$., $\$ 2.50$.

Rhododendrons, $\$ 1.25$.

\section{OTHER ORNAMENTALS.}

Azalea Mollis, 75c.

Magnolia Soulangeana-White and pink, $\$ 3.00$.

Magnolia Stellata, $\$ 2.50$.

Japanese Maples-

Golden Leaf, $\$ 1.50$.

Cut Leaf, \$1.50.

Purple Leaf, $\$ 1.50$.

Blood Leaf, \$1.50.

\section{HEDGE PLANTS.}

\section{California Privet-}

2 -year-old plants, $50 \mathrm{c}$ doz., $\$ 4.00$ per 100.

3 -year-old plants, 75 c dozen, $\$ 6.00$ per 100.

Dwarf Barberry- $\$ 1.25$ doz., $\$ 10.00$ per 100. 


\section{HARDY SHRUBS.}

Rose of Sharon-Double, pink, white and purple, $50 \mathrm{c}$ each.

Sweet-Scented Shrub - Flowers fragrant, like strawberries, $35 \mathrm{c}$ each.

Deutzia, Pride of Rochester-Double white flowers, $50 \mathrm{c}$ each.

Burning Bush - Scarlet pods in autumn, 50 c each.

Pearl Bush-Useful for cutflowers, white, $50 \mathrm{c}$ each.

Golden Bell - Bright, yellow flowers in April, 35c each.

Snowball Hydrangea-White flowers for three months, $50 \mathrm{c}$ each.

Hydrangea Grandiflora-White, gradually changing to rose, flowers remain in good condition for weeks, $35 \mathrm{c}$ each.

Lilac-White, fragrant flowers, 35 c each.

Lilac-Purple, fragrant flowers, 35c each.

Bridal Wreath-White, double flowers in May, 35c each.

Crimson Spirea-24 inches high, blooms spring and fall, $35 \mathrm{c}$ each.

Spirea V. H. - A complete fountain of white when in bloom. 35c each.

Snowball, Japanese - White flowers in June, 50 c each.

Weigelia E. R.-Crimson flowers, 35c ea.

Weigelia D.-Pink flowers, 35 c each.

\section{HARDY CLIMBING PLANTS.}

Boston Ivy-Clings to the wall, no flowers, $25 \mathrm{c}$ each.

Dutchman's Pipe - Small brown flowers, rapid growth, $50 \mathrm{c}$ each.

Honeysuckle-Monthly Fragrant or Dutch, constant bloomer, $25 \mathrm{c}$ each.

Honeysuckle-Halleana, nearly evergreen, fragrant, white flowers, $25 \mathrm{c}$ each.

Honeysuckle - Scarlet Trumpet, scarlet flowers, no odor, $25 \mathrm{c}$ each.

Kudzu Vine - Pea-shaped, rosy flowers, rapid grower, $35 \mathrm{c}$ each.

Clematis, Henryi-Large, white flowers, $50 \mathrm{c}$ each.

Clematis Jackmanii - Violet-purple, $50 \mathrm{c}$ each.

Clematis, Paniculata - Small, white, fragrant flowers, $25 \mathrm{c}$ each.

Wisteria, Purple-50 c each.

Wisteria, White- $50 \mathrm{c}$ each.

\section{HARDY PERENNIAL PLANTS.}

15 c each, $\$ 1.50$ per dozen.

Columbine-Assorted colors.

Coreopsis-Yellow flowers.

Larkspur-Blue shades.

Sweet William-Various colors.

Pinks-Assorted colors.
Plantain Lily - Broad leaves, lilac flowers. Hollyhocks-Assorted colors.

Iris, German-Assorted colors.

Japan Iris-Assorted colors, 25c each.

Phlox-Pink, White, Rose, Salmon, White with Pink Eye.

Golden Glow-Bright yellow flowers.

\section{ORNAMENTAL GRASS.}

25 c each, $\$ 2.50$ dozen.

Zena, Striped-Foliage marked crosswise, with bands of white and green.

Hardy Bamboo-Four to six feet tall.

Pampas Grass - Three to five feet high; feathery, purplish plumes.

Ribbon Grass-Narrow, dark green leaves, with silver mid-rib.

\section{ROSES. (Hybrid Perpetuals.)}

Anne De Diesbach-Fragrant, carmine.

Coquette De Alps-White, tinged Pink.

Clio-Flesh color.

Frau Karl Druscki-Snow white.

General Jacqueminot-Brilliant crimson.

Magna Charta-Bright, rosy pink.

Madam Gabriel Luizet - Soft pink, with flesh center.

Marshall P. Wilder-Full, deep red.

Mrs. John Lang-Pink, beautiful form.

Prince Camile De Rohan-Dark, velvet, crimson.

Paul Neyron-Deep pink.

Ulrich Brunner-Bright cerise-red.

Gruss-an-teplitz-Rich scarlet.

Hardy, Dormant Plants, Two Years Old.

30 c each, $\$ 3.00$ dozen.

\section{CLIMBING ROSES.}

Crimson Rambler - A rapid grower.

Dorothy Perkins-A beautiful pink.

Lady Gay - Flower opens pink, almost white when mature.

Two-Year-Old Plants.

$35 \mathrm{c}$ each, $\$ 3.50$ dozen.

\section{PREMIUMS ON PLANT ORDERS.}

These premium rates apply only to plants and trees at price per single plant. Do not apply on orders for special offers or at dozen rates.

\section{On Orders Amounting to}

$\$ 3.00$ select plants to the value of $\$ 0.30$ 5.00

$10.00 \quad$. $\quad$. $\quad$. $\quad$. $\quad 1.00$

And for each dollar above $\$ 10.00$, select extra plants to the value of 20 cents.

\section{WE DO NOT REPLACE TREES AT THE PRICES QUOTED.}

As a rule we can ship your order within three days after receiving it, but during the spring rush, orders are taken in rotation, and it is sometimes a week before we can ship. 